\title{
Role of vagus nerve in gastroduodenal adaptation and cytoprotection
}

\author{
Oksana Sulaieva, Natalia Obraztsova \\ Department of Histology, Cytology and Embryology, M. Gorky Donetsk National Medical University, Ukraine
}

Email address:

oksana.sulaieva@gmail.com (O. Sulaieva)

To cite this article:

Oksana Sulaieva, Natalia Obraztsova. Role of Vagus Nerve in Gastroduodenal Adaptation and Cytoprotection. American Journal of Clinical and Experimental Medicine. Vol. 2, No. 2, 2014, pp. 22-27. doi: 10.11648/j.ajcem.20140202.13

\begin{abstract}
Objective: In this review we focused on understanding the cause-and-effect relationships of gastroduodenal pathology aiming to clarify the role of vagus nerve. Results: The spectrum of vagus nerve biological effects in gastroduodenal area is related to its numerous targets and a wide range of its receptors. A variety of vagus nerve effects are related to the broad expression of cholinergic receptors on the target cells: smooth muscle cells, covering and glandular epithelium of stomach and duodenum, myofibroblasts and mast cells, vascular endothelium, intramural ganglion neurons, endocrine cells, platelets and blood leukocytes. In this paper, we discussed the following issues: 1) role of sensory nerve endings in the vagal reflex regulation; 2) impact of gastrin and leptin on vagal afferentation; 3) targets of vagus efferent nerves; 4) the role of acetylcholine in regulation of functional activity of oxyntic cells; 5) relationship of vagus efferents with enteroendocrine cells; 6) the role of vagus nerve in realization of compensatory and adaptive reactions in gastroduodenal area. Conclusion: Vagus nerve is one of the key regulators of mucosal activity and blood supply, modulating adaptive reactions and maintaining the gastrointestinal barrier.
\end{abstract}

Keywords: Gastroduodenal Area, Vagus Nerve, Afferent and Efferent Nerves

Gastrointestinal pathology takes one of the leading places in the morbidity rate. A long-time comprehensive study has helped in determining the spectrum of exogenous and endogenous pathological factors that are associated with gastroduodenal pathology. It can be stated, that issues of digestive system regulation has been in focus of scientist for more than two centuries [1]. Nevertheless, pathogenesis of such disorders as gastroesophageal reflux disease, gastritis, peptic ulcer disease, duodenitis is still not clear.

Structural homeostasis and functions of gastroduodenal area (GDA) are controlled by neural link, specific gastrointestinal regulatory peptides that have endo-, paraand neuroendocrine effects; and local factors - cytokines, growth factors, nitric oxide, etc. [2-3]. These regulatory molecules are produced by various cells of the mucosa and neurons of intramural ganglia. The auto-regulation, synchronicity, sequence, integration and protection of digestive organs are achieved due to the potentiation and modulation of these molecules [4, 5]. At the same time there is a hierarchy of GDA regulatory mechanisms, due to which any disorder automatically activates higher levels of management and control, that's why neuro-endocrine control goes on the forefront $[3,6]$. Initially, the centers of autonomic nervous systems are activated that leads us to the aim of this review to fully analyze the structural organization of the parasympathetic innervation and role of vagus nerve in the pathology and compensatory-adaptive processes in GDA.

Vagus nerve dependent gastric reflexes are mediated through vagal afferent fibers synapsing upon neurons of the nucleus tractus solitarius which, in turn, modulates the preganglionic parasympathetic dorsal motor nucleus within the medullary dorsal vagal complex [7]. These centers establish connection with the different parts of hypothalamus and cerebral cortex, providing the integration of metabolic control mechanisms, water-salt metabolism, digestion, and hemodynamics [6]. Vagal efferents are associated with neurons of the intramural ganglia in submucosa and musclularis externa, controlling contraction of smooth myocytes and secretion of glandular cells.

We focused on understanding the cause-and-effect relationships of gastroduodenal pathology and want to clarify the role of vagus nerve. It is, however, important to start with physiology of digestive process, which includes three phases: cerebral, gastric and intestinal. Regulation of secretion includes the cerebral phase that is mainly vagal with 
participation of some hormones. Experiments with false feeding, similar to the Pavlov's classic experiment, have shown that, in addition to the vagus nerve stimulation, the level of several gastric hormones was significantly increased. This set of regulators includes gastrin, cholecystokinin, histamine, gastrin-releasing peptide, ghrelin, etc. [8]. Also was observed a slight increase of some inhibitory hormones, such as pancreatic polypeptide YY (PYY), leptin, and secretin in the blood plasma [9]. The intensity of cerebral phase depends on multiple settings: activation of conditioned reflexes by the sense of smell and taste receptors of the tongue, and mechanical stimulation of the stomach and intestine chemoreceptors. Afferents activation of the gastroduodenal mucosa leads to the switching on of local (intramural) and vago-vagal (or extramural) reflexes that impact on the motility, mucus and secretion of the digestive juices and cytoprotection in GDA [10]. However, what signals determine the activation of afferent nerve fibers, and how does local protective system responses against the effects of aggressive exogenous and endogenous factors? Little is known. This explains the interest in structure and functional role of sensory nerve endings.

\section{Characteristics of Sensory Nerve Endings in Gastroduodenal Area}

Majority of GDA afferent nerve fibers is presented by dendrites of vagus sensory neurons and the intramural ganglia nerve cells. According to the modality these nerve endings were divided into two key types: mechanoreceptors and chemoreceptors [11]. Several authors also distinguish nociceptors, associated with the vascular wall. Today three types of mechanoreceptors are described, two of which starts from smooth muscle cells in the wall of gastrointestinal tract. The first type of receptors is similar to the lamellar bodies and is linked to myenteric ganglia. The terminal endings of the lamellar bodies are oriented parallel to the muscle layers and situated on the periphery of the ganglion - between neurons and smooth muscle cells, bound to them by glial cells. This morphological finding formed the assumption that this type of mechanoreceptors provides tension transduction when the muscular layer is stretching. Receptor field from each of the afferent neuron (ganglion) corresponds to a specific area of muscular layer, although described as overlapping fields of receptor neurons from different ganglia [12].

The second type of vagal afferents (intramural "rays") is different from the first by localization and structure. These endings are formed by long terminal dendrites, interconnected by short collaterals, and situated on the surface of smooth muscle cells. Separate terminal dendritesare linked to interstitial Cajal cells [13] (also known as interstitial myofibroblasts) in muscular layer, and by processes form a network that is oriented parallel to the bundles of smooth muscle cells. Apparently, these nerve endings perceive and transmit information about wall stretching. It is believed that intramuscular "rays" are accompanied by supporting cells, and join together with the nerve endings in the receptor unit, thus form a receptor network together with interstitial Cajal cells. Mice with genetic mutation in Cajal cells have shown that vagal innervation was disabled due to the "switching off" of intramuscular mechanoreceptors [14]. The use of neural tracers, associated only with the afferent nerve fibers of the digestive tract, has demonstrated that the highest density of lamellar nerve endings occurs in the stomach antrum and body, as well as in the proximal section of duodenum [12]. The quantity of these endings was much lower in the fundic and cardiac areas, around the gastroduodenal sphincter and distal colon. Intramuscular nerve endings predominate in the upper part of stomach and in the sphincter (mainly circular layer); a few can be found in the duodenum.

The third type of vagus nerve mechanoreceptors was only identified by electrophysiological methods, thus the structure has not been established yet. This kind of receptors is located in the mucosa and is a rapidly adapting type opposed to the slowly adapting muscle afferents. Reduction of the mechanoreceptors activity is observed due to the decreasing of chyme volume, which is limiting afferentation to the vagus centers.

First chemoreceptors were described in the GDAmucosa, although the vagal origin of it has not been established yet [11]. Using of anterograde tracers has recently allowed authors to prove their connection with the vagus. These vagal endings widely branch out in the gastric lamina propria and around intestinal crypts, and even in the duodenal villi, where they interact with subepithelial myofibroblast network. In addition, vagus sensory nerve endings were found near enterochromaffin cells of gastric and intestinal epithelium. In this connection it was suggested that myofibroblast network can form supporting and trophic structures for the arrangement of vagal afferents in the mucosa of gastrointestinal tract [12], like the interstitial Cajal cells in the muscularis externa. Thus, tight relationship between the vagus nerve fibers and the myofibroblast system opens up new prospects for researching the role of vagal afferents in the management of reparative processes, the other way around - the role of myofibroblasts in the development of denervation in the GDA mucosa under unlcerogenesis. Also it was proved, that vagal afferents contact with mast cells in the intestinal villi. This links determine formation of a feedback loop between microcirculation and neural control of GDA.

\section{Role of Afferent Nerves in Gastroduodenal Adaptation}

Under physiological conditions the most important and powerful activator of vagus nerve afferents is the increased concentration of $\mathrm{H}^{+}$ions. The increase of $\mathrm{H}^{+}$concentration in the gastric mucosa and afferent stimulation occurs in case of: a) damage of mucus-bicarbonate barrier and 
surface epithelium continuity; b) parietal cells alteration; c) microcirculation disorders [15].

One more significant element in the regulation of afferent sensitivity is a local endocrine system. Hormones can both directly and indirectly affect the afferentation. The direct effect is caused by the spatial closeness of chemoreceptors and enterochromaffin cells in the GDA mucosa. An example of such modulation can be a ghrelin recently isolated gastric peptide hormone that modulates neuro-visceral connection [7, 16]. Ghrelin, an orexigenic hormone secreted from the stomach, was soon after its discovery hypothesized to be a prokinetic agent, due to its homology to the motilin. Investigation of ghrelin physiology has demonstrated that ghrelin is secreted by gastric endocrine cells into the bloodstream, providing local effects by modulating the information up-flow through the vagus nerve to the brain stem nuclei and hypothalamus [17]. Additionally to this ghrelin may also stimulate the production of growth hormone. The expression and transport of ghrelin receptors has been documented for the afferent vagus nerve, and functional studies have confirmed that vagal pathways are integral to ghrelin-induced stimulation of gastric motility [18]. Combined stimulation of mechanosensitive gastroesophageal afferents and ghrelin injections allowed to find out that the direct effect of ghrelin was hidden in the decreasing afferent response to the stretching. Ghrelin inhibits vagal afferents activation, thus damage of vagus nerve afferents inhibits the appetitestimulating effects of ghrelin. Ghrelin not only increases a hydrochloric acid secretion, but also forms a positive feedback loop in the brain-gut axis, stimulating appetite, and participating in the formation of feeding behavior [16]. Overproduction of ghrelin decreases vagus afferentation that blocks formation of satiety during the stomach filling and stretching. The experimental studies, using ghrelin and ghrelin receptor agonists, confirmed this hypothesis, suggesting a therapeutic potential for the ghrelin receptor in the treatment of gastrointestinal motility disorders. Additionally to this effect, ghrelin acts centrally to increase mid-brain dopaminergic neurons activity, amplifies dopamine signaling and protect against neurotoxin-induced dopamine cell death in the mouse substantia nigra pars compacta [18]. In addition, ghrelin inhibits the lipopolysaccharide (LPS)-induced release of proinflammatory cytokines from peripheral macrophages and T-cell [19].

On the other hand there are another peptides with the opposite effect on vagal afferentation, like leptin, peptide YY, oxyntomodulin, glucagon-like peptide and amylin, that are released from endocrine cells of gut and pancreas [7]. As example gastric vagal afferents can be modulated by leptin, which is influenced by food intake status. In turn, energy intake is strongly influenced by vagal afferent signals from the stomach, and is also modulated by leptin. Interestingly, that leptin may be secreted from gastric epithelial cells [20]. Epithelial cells expressing leptin were found close to vagal mucosal endings. Firstly, it was shown that leptin potentiated vagal mucosal afferent responses to tactile stimuli. This conclusion was obtained during in vitro preparation and was used to determine the functional effects of leptin on gastric vagal afferents. The stimulating effect of leptin on gastric mucosal vagal afferents is mediated by phospholipase Cdependent activation of transient receptor potential channels. So, in vitro leptin potentiated vagal mucosal afferent responses to tactile stimuli. But next in vivo investigation has demonstrated that the effect of leptin on gastric vagal afferent excitability is dynamic and related to the feeding state. Surprisingly, after fasting or diet-induced obesity, potentiation of mucosal afferents by leptin was lost and leptin receptors expression reduced in the cell bodies of gastric mucosal afferents. These effects in diet-induced obese mice were accompanied by a reduction of vagal afferents density in the gastric mucosa. In striking contrast, after fasting or diet-induced obesity, leptin inhibited response to stretching in mechanoreceptors. The inhibitory effect on gastric afferents was mediated through phosphatidylinositol 3-kinase-dependent activation of calcium-activated potassium channels. These data suggest that in obesity, leptin actually reduces responses to gastric distension following food intake.

One more issue should be taken in attention is that increased afferentation is observed under the GDA mucosa inflammation, which is accompanied by enhanced production of inflammatory mediators, cytokines and growth factors [21]. Besides the induction of reflex control and feeding behavior modulation, vagal afferents are involved in the local system regulation, maintaining the homeostasis and GDA protection [22]. Thus, experiments have shown that capsaicin-induced deafferentation or vagotomy supports gastric and duodenal mucosa alteration under chronic ulcers [7]. In addition, the afferent nerve shutdown is accompanied by a decrease of adaptive reactions in microvascular circulation and mucusbicarbonate barrier (protection). So toxic agents diffusion (including hydrochloric acid) induces a rapid increase of the local blood flow due to the afferent nerves stimulation [10]. This effect is caused by the afferent releasing calcitonin gene-related peptide (CGRP) and substance $\mathrm{P}$ in the area of arterioles and so resulting in vasodilation. The releasing of biologically active substances from nerve afferents provides gastro-protection by increasing blood flow and reducing acid secretion. This effect is associated with stimulation of CGRP receptors type 1 of smooth muscle cells and arteriole endothelium, which leads to activation of ATP-sensitive $\mathrm{K}^{+}$channels, increased production of nitric oxide and prostaglandins [23]. Similarly, afferent activation in duodenum over the low luminal $\mathrm{pH}$ leads to the inclusion of local reflexes and serotonin release, which induces increased $\mathrm{HCO}_{3}{ }^{-}$secretion.

The change in afferent intensity and modality through the vagus sensory fibers alters the central regulative mechanisms of the gastrointestinal tract and the balance between sympathetic and parasympathetic autonomic nervous system activity. In case of an inappropriate 
stimulation under the inflammation and/or damage afferent signals reach CNS via the sensory fibers and are received as nociceptive. This is accompanied by the impulse conduction along the vagal efferent and sympathetic adrenergic nerve fibers. Vagus efferents activate $M$ cholinergic receptors and stimulate motility of the GDA, whereas activation of the sympathetic fibers shuts it down [11]. Terminals of adrenergic neurons inhibit activity of intramural cholinergic neurons, and smooth muscle cells hyperpolarization is provided via $\beta$-adrenergic receptors.

\section{Vagus Efferents Control Acid Secretion by Direct Stimulation and Negative Feedback System}

Vagus nerve is able to influence the gastric-duodenal motility and secretory activity via direct and indirect mechanisms. The direct contacts of the efferent vagal nerve were found on the following targets: a) smooth muscle cells; b) gastric parietal cells; c) G-cells of the pyloric glands and other enterochromaffin cells. The highest density of the vagus efferent nerve fibers was found in the muscle layer. There was described the synaptic contact formation, which includes following combinations: a) vagal ending + smooth muscle cell + intramural ganglion efferents; b) vagal efferents + intestinal cells of Cajal + smooth muscle cells [24]. In this case, quite a few mediators, produced by intramural ganglia neurons, can make functional links with cholinergic endings, so are: serotonin, bombesin, VIP, etc., as well as produced by interstitial cells (myofibroblasts) nitric oxide, prostaglandins, growth factors [13]. Apparently, change of these substances release is associated with gastrointestinal motility disorders under the divergent vagal control. The greatest interest for clinicians and pathophysiologists is the vagus nerve effects on mucosa, which in its turn creates the base of pathological and sanogenetic processes, defining ulcerogenesis or repair after damage.

An overactive vagus is considered to be one of the main "causer" in the increased acid secretion and progressive damage of gastroduodenal mucosa. Is this really so? To answer this question it is necessary to analyze the mechanisms of acetylcholine (ACh), as the main vagal neurotransmitter, influence on the parietal cells. The stimulating effect of $\mathrm{ACh}$ on the parietal cells is carried through $\mathrm{M}_{3}$ muscarinic cholinoreceptors on the basolateral membrane. Via a $\mathrm{G}_{\mathrm{q}}$-protein $\mathrm{ACh}$ activates phospholipase $\mathrm{C}$, with the formation of inositol-1,4,5-triphosphate (I3F) and diacylglycerol (DAG). I3F connects with glycosylated receptor protein $\mathrm{P} 400$, which is a $\mathrm{Ca}^{2+}$ pump that increases the level of intracellular $\mathrm{Ca}^{2+}$. The last one causes stimulation of $\mathrm{Ca}^{2+}$-dependent protein kinase $\mathrm{C}$, phosphorylation of effector molecules, increase expression of membrane transporters and pumps - first of all, a proton pump $\left(\mathrm{H}^{+}-\mathrm{K}^{+}-\mathrm{ATP}\right)$. An important effect of $\mathrm{ACh}$ is the activation of $\mathrm{Na}^{+}-\mathrm{K}^{+}$-ATPase on the basolateral surface of the parietal cells, which provides high level of potassium inside the cell and increases the permeability for $\mathrm{Ca}^{2+}$.

However, initiation of acid secretion mechanisms, is accompanied with simultaneous activation of restricting system for $\mathrm{H}^{+}$secretion and stimulates cytoprotection. This phenomenon is associated with the DAG hydrolysis that results in the release of arachidonic acid, which is metabolized under physiological conditions via the cyclooxygenase pathway to prostaglandin $\mathrm{E}_{2}\left(\mathrm{PGE}_{2}\right)$. The last one is a direct antagonist of histamine $\mathrm{H}_{2}$-receptors on parietal cells. Furthermore, $\mathrm{PGE}_{2}$ can bind to $\alpha$-regulatory subunit of adenylate cyclase, inducing the antisecretory effect. This way, the ACh-stimulation under physiological conditions is accompanied by increased production of $\mathrm{PGE}_{2}$, limiting excessive acid production [4].

Protective effects of $\mathrm{PGE}_{2}$ are connected with boosted microcirculation, increase of mucus secretion and bicarbonate transport through epithelial cells. In the ECLand G-cells prostaglandins stimulate cAMP formation, which in its turn causes reduction of $\mathrm{Ca}^{2+}$ influx into the cell and decrease calcium cytoplasmic level. All these result in reduced histamine and gastrin secretion. The only exception is the activation of 5-lipoxygenase (5-LOX) pathway of arachidonic acid metabolism under ischemia and oxidative stress. In this case leukotrienes are produced. 5-LOX activation in gastric mucosa leads to the formation of leukotrienesC4 and $\mathrm{C5}$, which amplify acid secretion and inflammation development, especially after stimulation with histamine [35].

Additionally to direct control of parietal cells, ACh provides stimulating effect on the G-cells. Gastrin stimulates histamine releasing from endocrine and mast cells by histidine decarboxylase activation. Schematically, the sequence of processes in the gastric mucosa can be represented as follows: vagus nerve stimulation - ACh release - G-cells stimulation - increasing gastrin production - ECL-cells stimulation - increasing histamine production. In its turn histamine through $\mathrm{H}_{2}$ receptors increases production of hydrochloric acid by activation of adenylate cyclase, increasing cAMP, stimulation of cAMPdependent protein kinase $\mathrm{A}$, that induces the translocation of $\mathrm{H}^{+}-\mathrm{K}^{+}$-ATPase into the apical plasmolemma of parietal cells [19]. However, it should be mentioned that histamine is also able to activate $\mathrm{H}_{3}$-receptors of ECL-cells, and by this way inhibits its activity and production in autocrine manner. The autocrine regulative loop restricts the hydrochloric acid production and limits the effects of vagal stimulation, as well as ACh release. Moreover, effect of Ach is short-term because of rapid destruction by acetylcholine esterase.

\section{Role of Vagus Efferents in Gastroduodenal Mucosa Protection}

Vagus shows up in numerous biological ACh effects such as stimulation of mucus secretion, bicarbonate production, 
cells proliferation, modulation of the immune response. A variety of ACh effects are related to the broad expression of cholinergic receptors on the target cells: smooth muscle cells, covering and glandular epithelium of stomach and duodenum, myofibroblasts and mast cells, vascular endothelium, intramural ganglion neurons, endocrine cells, platelets and blood leukocytes. Stimulation of a broad spectrum of N- and $\mathrm{M}$-cholinergic receptors includes not only an activation of transmembrane ions flows (sodium, potassium, calcium) and mobilization of intracellular $\mathrm{Ca}^{2+}$, but also changes in the activity of tyrosine kinases, G-proteins and mitogenactivated protein kinase (MAPK) [11].

Additionally to acid secretion modulation, VN plays the important role in gastric and duodenal epithelium integrity, stimulating expression of occludin and $\mathrm{ZO}-1$ via $n \alpha 7 \mathrm{CR}$, and maintenance of mucus bicarbonate barrier [22]. The latter one is quite important as mucus layer builds a physical barrier preventing $\mathrm{H}$. pylori adhesion to epithelial cells. The mucus layer in the stomach includes the secreted mucins MUC5AC, produced from the superficial mucosa, and MUC6, produced from the gland mucosa. On the one hand $\mathrm{H}$. pylori can cause substantial change in gastric mucus gel integrity and loss of the polymeric structure of mucins due to secretion of proteases and lipases. Additionally to this $\mathrm{H}$. pylori can induce impairment of mucins expression in the gastric mucosa. It was shown that MUC6, normally associated with mucous cells of the gastric glands, is expressed in surface mucous cells of $\mathrm{H}$. pylori infected patients while the MUC5 component of surface mucous cells decreases. It has have reported that MUC1 turnover and level are also decreased upon $H$. pylori infection in mice [1]. However, on the other hand, maintaining of normal pattern of mucins secretion in host limits $H$. pylory infection. Activation of MUC1 expression under VN stimulation, prostaglandin $\mathrm{E}_{2}$ and $\mathrm{NO}$ releasing provides a protective barrier, which limits both acute and chronic colonization by H. pylori, limiting the inflammation induced by this infection [4]. Hence, direct and indirect VN impact on mucus bicarbonate barrier can be involved in host-specific effects, determining self-protection and HP proliferation, gene expression and virulence.

Due to ACh effects, vagus nerve is considered to be one of the key factors in regulation of adaptive and compensatory processes. Especially, this may be connected with the stimulating effects of $\mathrm{ACh}$ on production of prostaglandins and nitric oxide(NO) that are considered as the main cytoprotectors and regulators in GDA structural integration. It was shown that direct inhibition if endothelial and neural nitric oxide synthase (eNOS and nNOS) results in blood flow disruption, motility and secretory disorders of the gastrointestinal tract [23]. In this way, NO is also involved in the cell protection against radicals and cytotoxic agents, moreover, under oxidative stress. The protective nature of the increased $\mathrm{NO}$ and $\mathrm{PGE}_{2}$ production under vagal stimulation is caused by blood flow optimization in mucosa, increased mucus and bicarbonates production, as well as modulation of the immune cells activity in mucosa and decrease of the adhesive interactions between endothelium and leukocytes. Recent evidence suggests that the vagal innervation of the gastrointestinal tract plays a major role by controlling gastrointestinal immune activation. Indeed, vagus nerve electrical stimulation potently reduces gastrointestinal inflammation restoring gut homeostasis, whereas vagotomy has the reverse effect [21].

In conclusion, vagus nerve is one of the key factors in regulation of mucosal barrier and blood supply, providing realization of adaptive and compensatory processes.

\section{References}

[1] Konturek SJ, Konturek PC, Brozozowski T, et al. From nerves and hormones to bacteria in the stomach; Nobel prize for achievements in gastrology during last century. J Physiol Pharmacology 2005; 56, 507-530.

[2] Allen A, Flemstrom G. Gastroduodenal mucus bicarbonate barrier: protection against acid and pepsin. Am J Physiol Cell Physiol 2004; 288, 1-19.

[3] Blackshaw LA. Receptors and transmission in the brain-gut axis: potential for novel therapies.Am J Physiol Gastrointest Liver Physiol 2001; 281, 311-315.

[4] Gyires K. Gastric mucosal protection: from prostaglandins to gene-therapy. Curr Med Chem2005; 12, №2, 203-215.

[5] Calatayud S, Barrachina D, Esplugues JV. Nitric oxide: relation to integrity, injury, and healing of the gastric mucosa. Microsc Res Tech 2001; 53, №5, 325-335

[6] Gyires K, Nemeth J, Zadori ZS. Gastric mucosal protection and central nervous system. Curr Pharm Des 2013; 19, №1, 34-39.

[7] Kirkup AJ, Brunsden AM, Grundy D. Receptors and transmission in the brain-gut axis: Potential for novel therapies I. Receptors on visceral afferents. Am J Physiol Gastrointest Liver Physiol 2001; 280, 787-794.

[8] Cui G, Waldum HL. Physiological and clinical significance of enterochromaffin-like cell activation in the regulation of gastric acid secretion. World J of Gastroenterol 2007; 13, № 4, 493-496.

[9] Stengel A, Tache Y. Gastric peptides and their regulation of hunger and satiety. Curr. Gastroenterol Rep 2012; 14, №6, 480-488.

[10] Ko JK, Cho CH. Adaptive cytoprotection and the brain-gut axis. Digestion 2011; 83 Suppl 1, 19-24.

[11] Berthoud HR, Neuhuber WL. Functional and chemical anatomy of the afferent vagal system. Auton Neurosci 2000; $85,1-17$.

[12] Powley TL, Gilbert JM, Baronowsky E.A, et al. Vagal sensory innervation of the gastric sling muscle and antral wall: implications for gastro-esophageal reflux disease? Neurogastroenterol Motil 2012; 24, №10, 526-537.

[13] Kito Y. The functional role of intramuscular interstitial cells of Cajal in the stomach. J Smooth Muscle Res 2011; 47, №2, 47-53. 
[14] Ward SM, Sanders KM, Hirst GD. Role of interstitial cells of Cajal in neural control of gastrointestinal smooth muscles. Neurogastroenterol Motil 2004; 16, 112-117.

[15] Kang YM, Bielefeldt K, Gebhart GF. Sensitization of mechanosensitive gastric vagal afferent fibers in the rat by thermal and chemical stimuli and gastric ulcers. J Neurophysiol 2004; 91, 1981-1989.

[16] Cummings DE, Frayo RS, Marmonier C, et al. Plasma ghrelin levels and hunger scores in humans initiating meals voluntarily without time- and food-related cues. Am J Physiol Endocrinol Metab 2004; 287, 297-304.

[17] Page AJ, Slattery JA, Milte C. Ghrelin selectively reduces mechanosensitivity of upper gastrointestinal vagal afferents. Am J Physiol Gastrointest Liver Physiol 2007; 292, 13761384.

[18] Yang CG, Wang WG, Yan J, et al. Gastric motility in ghrelin receptor knockout mice. Mol Med Rep 2013; 7, №1, 83-88.

[19] Khan WI, Ghia JE. Gut hormones: emerging role in immune activation and inflammation. Clin Exp Immunol 2010; 161, №1, 19-27.

[20] Yarandi SS, Hebbar G, Sauer CG, et al. Diverse roles of leptin in the gastrointestinal tract: modulation of motility, absorption, growth, and inflammation. Nutrition 2011; 27, №3, 269-275.

[21] Lamb K, Kang YM, Gebhart GF, et al. Gastric inflammation triggers hypersensitivity to acid in awake rats. Gastroenterology 2003; 125, 1410-1418.

[22] Martin GR, Wallace JL. Gastrointestinal inflammation: a central component of mucosal defense and repair. Exp Biol Med (Maywood) 2006; 231, №2, 130-137.

[23] Takahashi T, Owyang C. Vagal control of nitric oxide and vasoactive intestinal polypeptide in the regulation of gastric relaxation in rat. J Physiol 2008; 484,481-492.

[24] Musara C, Vaillant C. Immunohistochemical studies of the enteric nervous system and interstitial cells of Cajal in the canine stomach. Onderstepoort J Vet Res 2013; 80, №1, 1-4. 